\title{
Portal vein catheterization and selective angiography in diagnosis of total anomalous pulmonary venous connexion
}

\author{
Michael Tynan, D. Behrendt, W. Urquhart, and G. R. Graham \\ From the Cardiology Department, Newcastle General Hospital, Westgate Road, Newcastle upon Tyne; and \\ The Thoracic Unit, The Hospital for Sick Children, Great Ormond Street, London
}

\begin{abstract}
Portal venous system catheterization via the umbilical vein is easily accomplished in the neonatal period. Using this approach, in combination with conventional cardiac catheterization, a precise diagnosis of subdiaphragmatic total anomalous pulmonary venous connexion can be made. Selective angiography in the common pulmonary vein can be performed using a small dose of contrast medium. This delineates the anatomical distribution of the pulmonary veins and site or sites of pulmonary venous obstruction more accurately than recirculation angiography. Direct pulmonary venous angiography eliminates the need for the injection of contrast medium into the arterial phase of the pulmonary circulation. This, together with the low dose of contrast medium needed, may lower the risk of investigation in these patients.
\end{abstract}

Umbilical vein catheterization has been proposed as a method of cardiac catheterization in the neonatal infant (Linde et al., 1966; Sapin, Linde, and Emmanouilides, 1963) and its value has been suggested in the diagnosis of subdiaphragmatic total anomalous pulmonary venous connexion (Airaghi, Calo, and Soresi, 1969; Sneed, 1972). In this paper we present haemodynamic and angiocardiographic data from two patients with subdiaphragmatic total anomalous pulmonary venous connexion; in one case the connexion was to the inferior vena cava, in the second the connexion was to the portal vein.

\section{Methods and case reports}

Two infants were investigated, cardiac catheterization was carried out via the right saphenous vein in the groin and the portal system was catheterized via the umbilical vein, using an incision $\mathrm{I} \mathrm{cm}$ above the umbilicus. Portal system catheterization was undertaken when the haemodynamic data strongly suggested that obstructed subdiaphragmatic anomalous pulmonary venous connexion was present. Conventional 5 French woven 'dacron' catheters were used, pressures were measured in peak expiration using strain gauge transducers with a direct writing recorder, reference zero at midthorax. Oxygen saturations were measured using haemoreflection oximetry.

In both cases the history, physical signs, plain chest $x$-ray, and electrocardiogram suggested obstructed total Received 20 May 1974. anomalous pulmonary venous connexion. Cardiac catheterization was carried out as an emergency in both cases. Case I was a female infant, aged 5 days, weighing $2.9 \mathrm{~kg}$. Case 2 was a male infant, aged II days, weighing $3.4 \mathrm{~kg}$. In both cases left ventricular angiography was performed in addition to the investigation of the pulmonary venous return.

Pulmonary artery angiography was performed in Case I using $7 \mathrm{ml}$ conray 480 , allowing the programme to run for 24 seconds. There was insufficient opacification of the pulmonary veins for an accurate diagnosis to be made, so pulmonary artery angiography was repeated after a period of artificial ventilation with I0O per cent oxygen. At this second investigation the common pulmonary vein was entered directly from the inferior vena cava, bradycardia ensued rapidly so no direct pulmonary venous angiogram was possible, only withdrawal pressures were recorded (Fig. 2); no sample was taken for oximetry. On the basis of the recirculation angiograms no operation was considered feasible and the patient died.

In Case 2 common pulmonary vein angiography was performed, $3 \mathrm{ml}$ 'cardio-conray' being used. A diagnosis of obstructed total anomalous pulmonary venous connexion to the portal vein with persistent ductus arteriosus was made. There was also a coarctation of the aorta on angiography, but there was no pressure gradient from the left ventricle to the descending aorta. A corrective operation was attempted including ligation of the ductus arteriosus. The patient died 72 hours after operation.

Necropsy confirmed the diagnosis in both cases. In Case 2, the left atrium to pulmonary vein anastomosis 
FIG. I Case I. Recirculation angiogram of the pulmonary venous system in total anomalous pulmonary venous connexion to inferior vena cava.

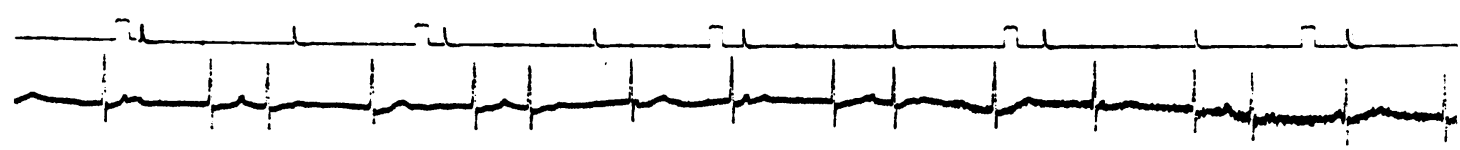

Anomalous Vein

IVC

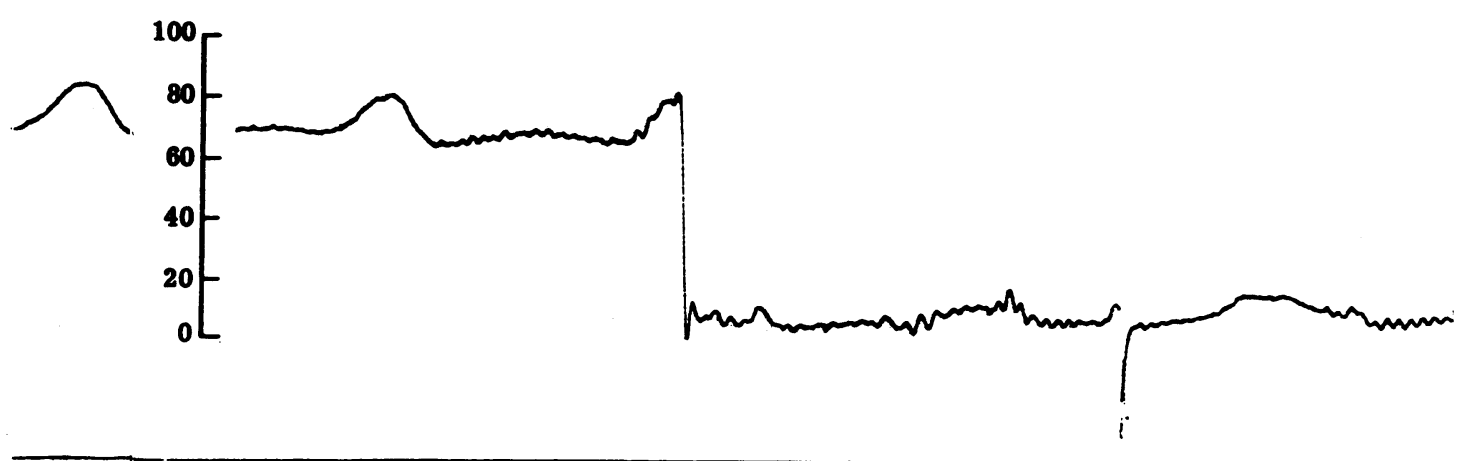

FIG. 2 Case I. Withdrawal pressure trace from the anomalous common pulmonary vein to inferior vena cava (IVC). Scale in $\mathrm{mmHg}$.

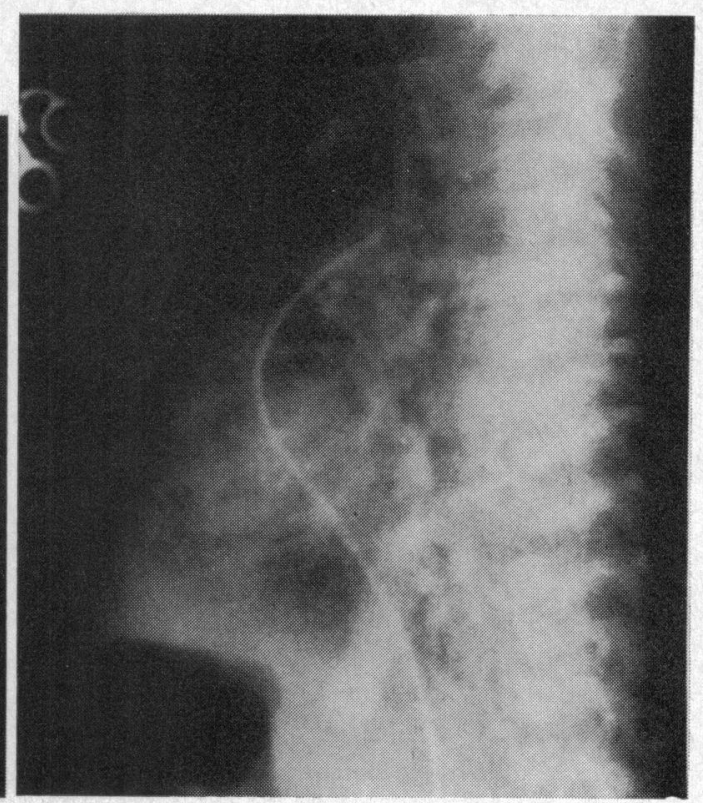



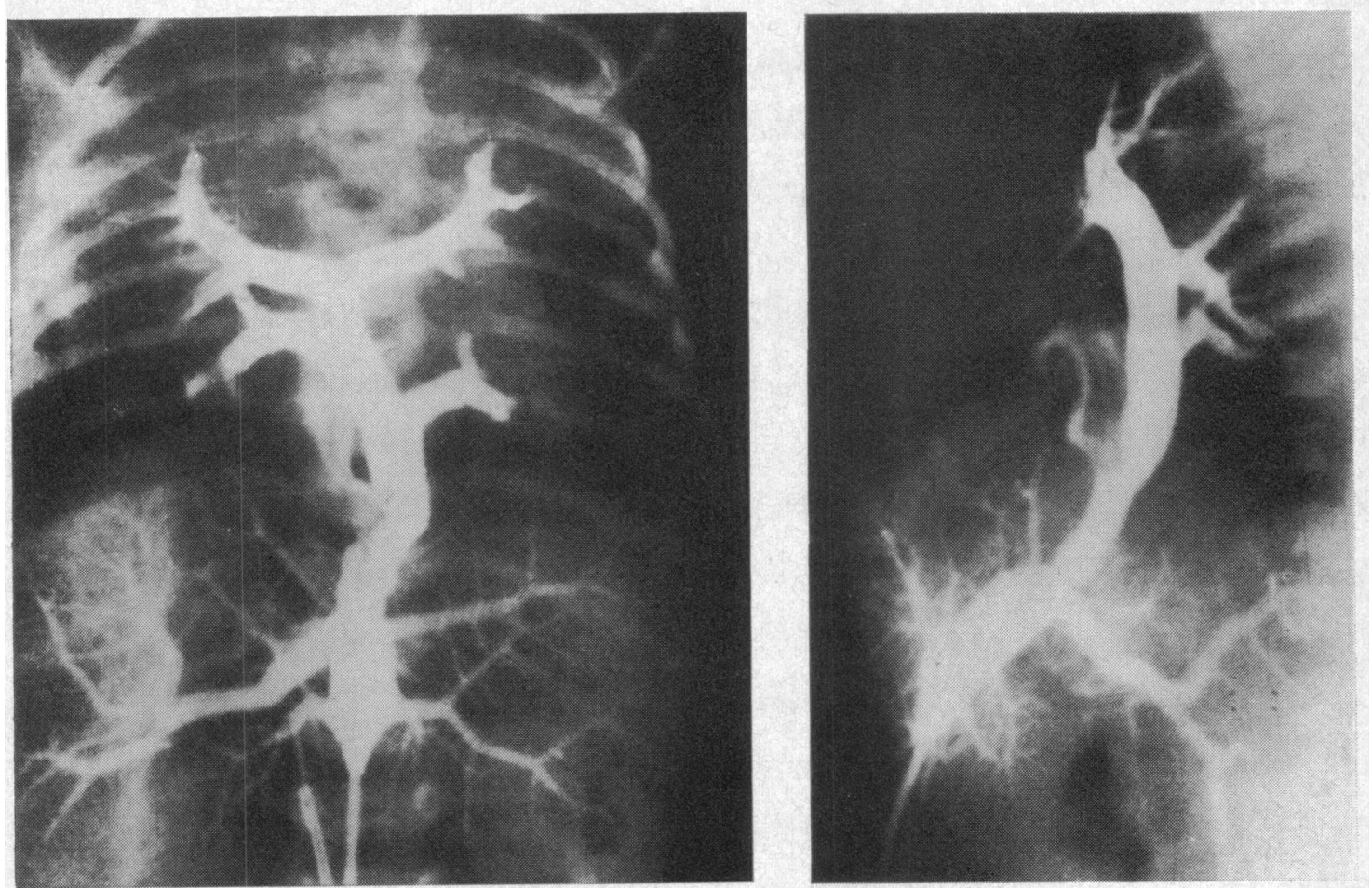

FIG. 3 Case 2. Selective direct common pulmonary venous angiogram in total anomalous pulmonary venous connexion to portal vein.

TABLE Cardiac catheterization data

\begin{tabular}{|c|c|c|c|c|c|c|}
\hline \multirow[b]{2}{*}{ Site } & \multicolumn{3}{|c|}{$\begin{array}{l}\text { Case I } \\
\text { (breathing } 100 \% \text { oxygen) }\end{array}$} & \multicolumn{3}{|c|}{$\begin{array}{l}\text { Case } 2 \\
\text { (breathing air) }\end{array}$} \\
\hline & $\mathrm{O}_{2} \%$ sat. & $\begin{array}{l}\text { Pressure (m) } \\
\text { phasic }\end{array}$ & mean & $\mathrm{O}_{2} \%$ sat. & $\begin{array}{l}\text { Pressure (mm } \\
\text { phasic }\end{array}$ & mean \\
\hline $\begin{array}{l}\text { Main pulmonary artery } \\
\text { Right ventricle } \\
\text { Superior vena cava } \\
\text { Right atrium } \\
\text { Inferior vena cava, high } \\
\text { Systemic artery } \\
\text { Left ventricle } \\
\text { Left atrium } \\
\text { Hepatic vein wedge } \\
\text { Portal vein } \\
\text { Common pulmonary vein }\end{array}$ & $\begin{array}{l}60 \\
50 \\
40 \\
68 \\
62 \\
68 \\
67 \\
64 \\
\frac{53}{6} \\
\end{array}$ & $\begin{array}{l}\text { I } 10 / 60 \\
120 / 10 \\
a=8, v=3 \\
75 / 50 \\
80 / 6 \\
a=7, v=2\end{array}$ & $\begin{array}{r}\text { I } \\
55 \\
\text { I } \\
4 \\
4 \\
80\end{array}$ & $\begin{array}{l}68 \\
62 \\
35 \\
37 \\
33 \\
34 \\
37 \\
- \\
60 \\
76 \\
99 \text { breathi }\end{array}$ & $\begin{array}{l}75 / 40 \\
84 / 7 \\
a=10, v=8 \\
60 / 45 \\
55 / 12 \\
a=12, v=9 \\
\end{array}$ & $\begin{array}{r}9 \\
38 \\
9 \\
12 \\
8 \\
22\end{array}$ \\
\hline
\end{tabular}



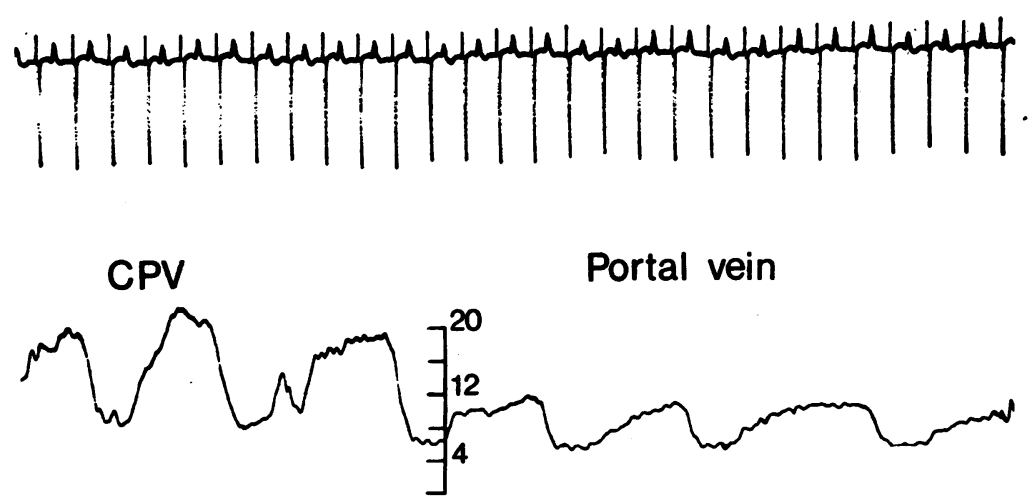

FIG. 4 Case 2. Withdrawal pressure trace from the common pulmonary vein to the portal vein in total anomalous pulmonary venous connexion to portal vein. Scale in mmHg. CPV= anomalous common pulmonary vein.

appeared adequate but the coarctation of the aorta, which had not been resected, appeared to have been more severe than the investigations suggested and was probably a haemodynamically significant one.

\section{Results}

The cardiac catheterization data are shown in the Table. In both cases the pulmonary arterial pressure was higher than the systemic arterial pressure. A right-to-left shunt was demonstrated at atrial level in both infants and a left-to-right shunt was suggested in Case I by the highest oxygen saturations being detected in the high inferior vena cava and right atrium. In Case 2 no evidence of a left-toright : shunt was found. The higher pulmonary arterial saturation in this case was found at the start of the procedure, the lower saturations were found later in the procedure.

In neither case was portal vein hypertension present nor was it suggested by the hepatic vein wedge pressures. In Case I portal vein catheterization showed no evidence of pulmonary venous blood in the portal system even though the patient was breathing 100 per cent oxygen. In Case 2, however, the portal vein had a higher oxygen saturation than the immediately preceding cardiac saturations, and moreover the pulmonary veins were demonstrated to be entering the portal vein; the pulmonary veins became fully saturated on breathing 100 per cent oxygen. The withdrawal trace from the common pulmonary vein to portal vein, shown in Fig. 4, suggested that the major obstruction to flow in the pulmonary venous system occurred at the junction of the pulmonary and portal veins. The withdrawal trace from common pulmonary vein to inferior vena cava (Fig. 2) in Case I showed that this junction was the sole site of obstruction to flow in the pulmonary veins in this patient. Both these observations were supported by the angiography (Fig. I and 2). Direct pulmonary venous angiography in Case 2 (Fig. 3) provided much better anatomical detail than the recirculation angiocardiography performed in Case I (Fig. I); in addition to this, significant flow is seen to be occurring through the ductus venosus to the right atrium in Case 2 (Fig. 3).

\section{Discussion}

Portal venous system catheterization via the umbilical vein can be performed easily in the neonatal infant and, if it is present, anomalous pulmonary venous connexion to the portal vein can be demonstrated haemodynamically. This is facilitated by the administration of Ioo per cent oxygen as the inspired gas. Manipulation of the catheter into the common pulmonary vein, in Case 2, presented no difficulty and angiograms performed at this site are self evidently better than those obtained by pulmonary artery injection.

They can be performed with a low dose of contrast medium, in this case less than I $\mathrm{ml}$ cardioconray per kg body weight. Using this method, not only are better angiograms obtained, but no contrast is injected into the arterial side of the pulmonary circulation, thus decreasing the risk of angiocardiography (Grainger, 1965, 1968). The differentiation between obstructed total anomalous pulmonary venous connexion to the portal system and other forms of obstructed anomalous pulmonary venous connexion, including drainage to the inferior vena cava, can be made using portal venous catheterization and angiography. This method should have an additional application in complete anatomical description of complex combinations where 
more than one site of connexion of anomalous pulmonary veins is suspected.

On a more speculative note, the fact that Case 2 had survived for II days and had obvious flow through the ductus venosus suggests that, though the major obstruction appears to be at the junction of the pulmonary and systemic veins, closure of the ductus venosus, with the consequent necessity for the pulmonary venous blood to traverse the hepatic venous bed, may cause a second, and fatal, source of obstruction in anomalous venous connexion to the portal vein.

\section{References}

Airaghi, M., Calo, S., and Soresi, E. (1969). Lo Sbocco anomalo totale delle vene polmonari nel seno venoso portale (Valore diagnostico della tensione di $\mathrm{O}_{2}$ ne sangue della vena ombelicale). Minerva Pediatrica, 21, 76.
Grainger, R. G. (1965). Complications of cardiovascular radiological investigations. British fournal of Radiology, 38, 201.

Grainger, R. G. (1968). Cardio-Conray - a new contrast medium formulation for angiocardiography and aortography. British fournal of Radiology, 41, 674.

Linde, L. M., Higashino, S. M., Berman, G., Sapin, S. O., and Emmanouilides, G. C. (1966). Umbilical vessel cardiac catheterization and angiocardiography. Circulation, 34,984 .

Sapin, S. O., Linde, L. M., and Emmanouilides, G. C. (1963). Umbilical vessel angiocardiography in the newborn infant. Pediatrics, 31, 946.

Sneed, R. C. (1972). Total anomalous pulmonary venous return: diagnosis by umbilical vessel catheterization. Southern Medical fournal, 65, II45.

Requests for reprints to Dr. Michael Tynan, Cardiology Department, Newcastle General Hospital, Westgate Road, Newcastle upon Tyne NE4 6BE. 